\title{
Sensing Properties of Nanocrystalline Silicon Carbide in Wet Condition
}

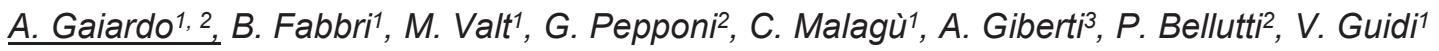 \\ 1 Department of Physics and Earth Science, University of Ferrara, Via Saragat 1/c, 44122 Ferrara, \\ Italy \\ ${ }^{2}$ MNF - Micro Nano Facility, Bruno Kessler Foundation, Via Sommarive 18, 38123 Trento, Italy \\ ${ }^{3}$ MIST E-R s.c.r.I., Via P. Gobetti 101, 40129 Bologna, Italy \\ Corresponding author's e-mail address: andrea.gaiardo@unife.it
}

\begin{abstract}
Silicon carbide is a well-known material with high thermal and chemical stability. In this work, we present an investigation on the chemoresistive properties of nanostructured Silicon Carbide (SiC). A commercially available nanopowder of silicon carbide was first purified and then its morphology, structure and thermal stability was characterized. Afterwards, the powder was mixed with suitable organic vehicles and screen-printed onto alumina substrates. SiC thick films were tested as chemoresistive gas sensors in thermo-activation mode. In this way, they were exposed to 13 gases belonging from different chemical classes. The sensing characterization showed that this semiconductor is an extremely selective functional material for the detection of sulphur dioxide $\left(\mathrm{SO}_{2}\right)$ at temperature higher than $600^{\circ} \mathrm{C}$, useful for harsh environments. Also, the sensing properties of SiC sensors were strongly improved by the presence of humidity. The sensing mechanism was also investigated and a possible interpretation has been proposed.
\end{abstract}

Key words: Chemoresistive gas sensors, nanostructured silicon carbide, sulphur dioxide, high selectivity, wet condition.

\section{Introduction}

Silicon carbide is a long-time known material, massively produced starting from 1890. Ceramics obtained by sintering $\mathrm{SiC}$ grains are very robust, then they find employment in car brakes, bullet-proof vests and in general in high endurance applications [1]. SiC thermal strength is also extraordinary, it holds stability even above $1000^{\circ} \mathrm{C}$, making it possible hightemperature applications ( $\mathrm{SiC}$ melting point is above $2500^{\circ} \mathrm{C}$ at 35 atm). Electronic applications of $\mathrm{SiC}$ are light-emitting diodes and high-temperature and/or high-voltage devices, as JFETs and MOSFETs rated at $1200 \mathrm{~V}$ [2].

Encouraged by the diverse fields in which SiC can be applied and the possibility to use it in form of a nanosized powder, we decided to investigate its possible chemoresistive properties for gas-sensing applications. There are no studies about chemoresistive properties of $\mathrm{SiC}$ nanoparticles at the best of our knowledge, apart from our previous study [3].

\section{Experimental}

First, commercial nanostructured SiC was purified by means of subsequent cycles of powder, then it was characterized from the morphological, thermal and structural point of view. These characterizations highlighted the high chemical purity and the nanometric size of the SiC powder (Fig. 1). Furthermore, the structural characterization showed the presence of one predominant crystalline phase in the $\mathrm{SiC}$ sample.

Afterwards, the material was screen-printed onto alumina substrates and tested as chemoresistive gas sensor. The electrical characterization of the SiC sensors was carried out in a test chamber by means of the flowthrough technique. SiC thick films were exposed to 13 different gases, and the responses of the sensors were investigated at operating temperatures ranging from $250{ }^{\circ} \mathrm{C}$ to $800^{\circ} \mathrm{C}$. The measurements have been performed both in dry and wet conditions.

The most interesting result was that $\mathrm{SiC}$ sensors proved to be insensitive to almost all gases analysed, while showed a significant response to sulphur dioxide in the concentration range of some ppm, in dry and in wet conditions (Fig. 2). 


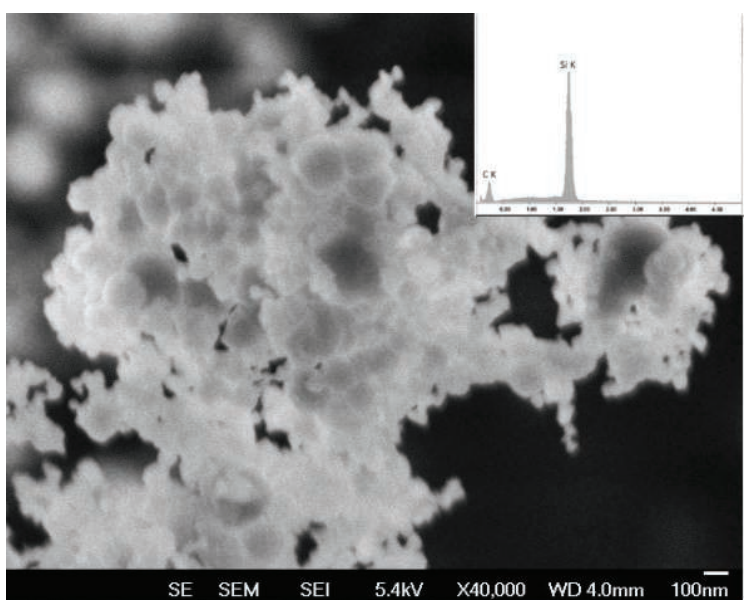

Fig. 1. SEM Image and EDX analysis of SiC nanopowder.

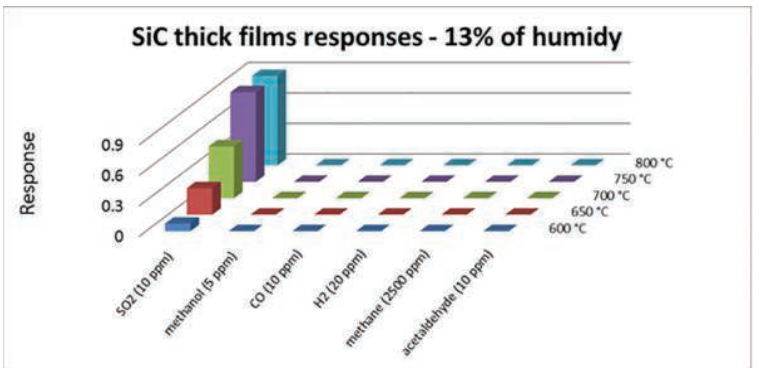

Fig. 2. The sensing selectivity of $\mathrm{SiC}$ films to $\mathrm{SO}_{2}$, in presence of humidity.

The presence of the humidity increased the stability and the response of the $\mathrm{SiC}$ films vs. $\mathrm{SO}_{2}$ (Fig. 3).

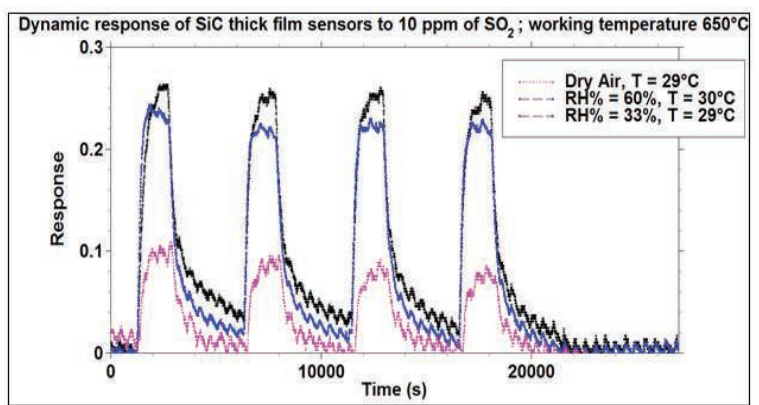

Fig. 3. Comparison of SiC gas sensing responses, vs. $\mathrm{SO}_{2}$, in dry air, $\mathrm{RH}=33 \%$ and $\mathrm{RH}=60 \%$.

The calibration curve of $\mathrm{SiC}$ thick film vs. $\mathrm{SO}_{2}$ concentrations highlighted a nonlinear correlation, similar to the trend of common metal oxides. The detection limit was about lower than $1 \mathrm{ppm}$ of $\mathrm{SO}_{2}$ (Fig. 4). Further investigations highlighted that the interesting sensing property of $\mathrm{SiC}$ films was due to the formation of a $\mathrm{SiO}_{2} / \mathrm{SiC}$ core shell, starting from a temperature of $600^{\circ} \mathrm{C}$ (Fig. 5).

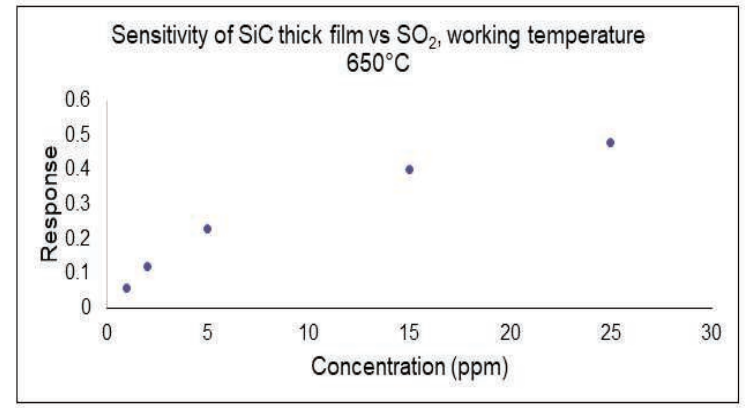

Fig. 4. Sensitivity of the SiC thick film vs. $\mathrm{SO}_{2}$ contrantions.

Indeed, the formation of this core shell increased the reactivity of $\mathrm{SiC}$ films.

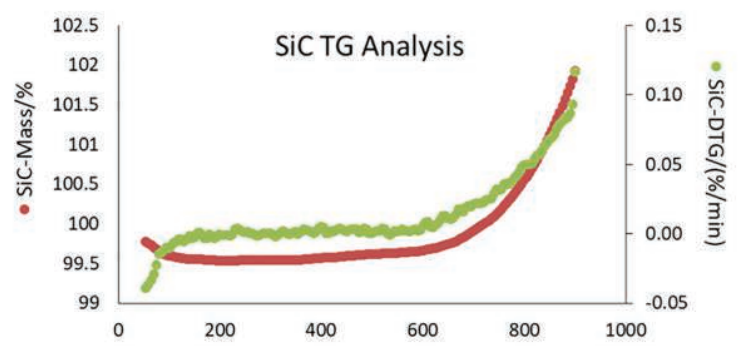

Fig. 5. XPS analysis of SiC nanopowder before the heat treatment (red line) and after 2 hours at $650^{\circ} \mathrm{C}$ (green line) and at $850^{\circ} \mathrm{C}$ (black line).

Finally, a gas sensing mechanism has been proposed. With this aim, exhaust gases of the reaction between $\mathrm{SO}_{2}$ and $\mathrm{SiC}$ films at $650^{\circ} \mathrm{C}$ were analysed by means of PTR-MS technique, to investigate the possible chemical reaction. Applications of such a sensor could span many fields, since sulphur dioxide plays an important role in air pollution, industrial processes and winemaking.

\section{References}

[1] W. Krenkel, F. Berndt, C/C-SiC composites for space applications and advanced friction systems, Materials Science and Engineering $A$, 412 (1-2), 177-181 (2005); doi: 10.1016/j.msea.2005.08.204.

[2] Yu, L.C., Dunne, G.T., Matocha, K.S., Cheung, K.P., Suehle, J.S., Sheng, K. Reliability issues of SiC MOSFETs: A technology for hightemperature environments, IEEE Transactions on Device and Materials Reliability, 10 (4), 418-426 (2010); doi: 10.1109/TDMR.2010.2077295.

[3] A. Gaiardo et al., Chemoresistive Gas Sensor based on SiC Thick Film: Possible Distinctive Sensing Properties between $\mathrm{H}_{2} \mathrm{~S}$ and $\mathrm{SO}_{2}$, Procedia Engineering, 168, 276-279 (2016); doi: 10.1016/j.proeng.2016.11.191 